\title{
Flexibility Evaluation of Hybrid WDM/TDM PONs
}

\author{
Abhishek Dixit, Bart Lannoo, Goutam Das, Didier Colle, Mario Pickavet, Piet Demeester \\ Department of Information Technology, Ghent University - IBBT, B-9050 Gent, Belgium \\ abhishek.dixit@intec.ugent.be
}

\begin{abstract}
A hybrid WDM/TDM passive optical network $(\mathrm{PON})$ is a promising candidate for next-generation optical access (NGOA) solutions. Several hybrid WDM/TDM PON architectures can be designed, each with a different degree of flexibility, going from fully static, over partially flexible to fully flexible architectures. A flexible architecture can serve several advantages, like energy efficiency, network migration and network extensibility. The more flexible architectures, however, are either more expensive, experience a higher power loss or are less secure. A question that arises is if a fully flexible architecture really needed. An important assessment parameter is the number of wavelengths required at a certain network load. In this paper, we introduce and compare two different flavors of flexibility based on the multicasting and switching functionality of different architectures. By exhaustive simulation, we study the gains of different variants of flexibility with different traffic models.
\end{abstract}

\section{INTRODUCTION}

Optical fiber networks are considered the most future-proof next-generation access (NGA) technologies to address the demands for ever increasing traffic volumes. Nowadays, the commonly deployed power splitter based time division multiplexing (TDM) passive optical networks (PON) are unable to provide the expected residential data rates by the year 2020 [1], and for that reason several next-generation optical access (NGOA) solutions are proposed and evaluated. Applying wavelength division multiplexing (WDM) to a PON is currently deployed by a few operators worldwide. However, a pure WDM PON offers a separate wavelength channel per user and hence provides no opportunity to share capacity among the users, and to use the network resources in a flexible way.

A hybrid WDM/TDM PON system combines the WDM domain with a TDM PON and can deliver both the benefits of an increased capacity delivered by WDM and the inherent capacity sharing of a TDM PON. Different hybrid WDM/TDM PON flavors are presented in [2]. The nextgeneration hybrid WDM/TDM PON can be either fully passive with arrayed waveguide gratings (AWGs) and/or power splitters at remote node 1 (RN1, e.g. at today's position of central office) or semi-passive with a switching element like a wavelength selective switch (WSS) at RN1. The architectures presented in [2] vary in terms of flexibility, cost, power consumption, and data security. We refer to 'flexibility' as the architectural capability to offer the same network (PON) performance with a smaller number of used wavelengths.
In this paper, we introduce and compare two different flavors of flexibility based on the functionality of the different architectures. While an AWG based hybrid WDM/TDM PON only offers what we call as "multi-casting flexibility"; the WSS based hybrid WDM/TDM PON offers both "multicasting" as well as "switching flexibility". Paper [3] presents a preliminary study of 'flexibility' in an AWG based hybrid WDM/TDM PON. In this paper, we extend the flexibility evaluation to study the gains of flexibility in both AWG and WSS based hybrid WDM/TDM PON. We also present the effect of traffic characteristics on the gains of flexibility. Section II provides a brief description of different flexible hybrid WDM/TDM PON architectures. In section III, different models and parameters considered for the flexibility evaluation are discussed. In section IV, simulation results for different traffic types are presented. Finally section V concludes the paper.

\section{HYBRID WDM/TDM PON ARCHITECTURES}

A general architecture of a (flexible) hybrid WDM/TDM PON is shown in Fig. 1, consisting of an optical line terminal (OLT) in the central office, an optical network unit (ONU) at the user side, and two remote nodes in between. In remote node 2 (RN2), a passive power splitter $(1: N)$ is installed, which means that RN1 is connected to $M$ TDM PON architectures. By varying RN1, different flavors of a hybrid WDM/TDM PON with a varying degree of flexibility can be obtained. Generally, flexibility can be differentiated in two types:

a) Flexibility due to multicasting functionality

b) Flexibility due to switching functionality

In architectures having multicasting functionality, one wavelength may be shared amongst many users. In this way, if the load of the users sharing the same wavelength is small, a new wavelength will not be allocated to them. Thus, the channel under-utilization is reduced as now necessarily one user will not be allocated one wavelength. This reduces wavelength usage and makes the architecture flexible. Multicasting functionality stems from using power splitters at the remote nodes. Due to the power splitter at RN2, there is always some degree of flexibility present in a hybrid WDM/TDM PON.

In architectures with switching functionality, the wavelength allocation is dynamic and according to the instantaneous traffic demand. By dynamic configuration of wavelengths, the users with high load can be allocated extra wavelengths and thus the general PON performance is improved. This will lead to a smaller probability that the network will be overloaded. We define the network overloading factor (NOF) as the probability of the network 


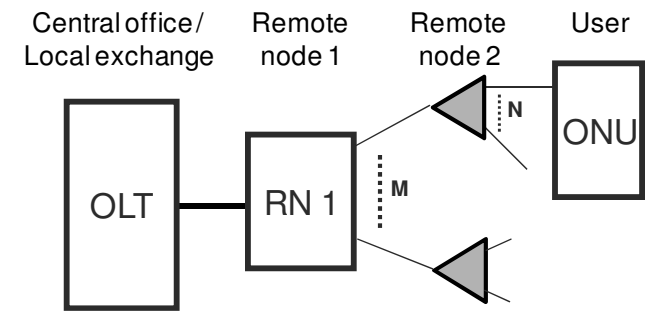

Figure 1: General hybrid WDM/TDM PON architecture

demand to exceed the network resources. A higher NOF will lead to higher delays in the network. The architectures with active components like WSS in RN1 offer "switching flexibility".

We now discuss various flavors of hybrid WDM/TDM PON with a varying degree of flexibility:

Fully Flexible Architectures: Table 1(a) provides an example of RN1 of a fully flexible architecture, which is referred to in the literature as broadcast-and-select $(\mathrm{B} \& \mathrm{~S})$ hybrid WDM/TDM PON. In a B\&S hybrid WDM/TDM PON, RN1 is a power splitter; all wavelengths are broadcast to all TDM PONs (or RN2s) and each ONU can choose any wavelength or time slot according to its need. This architecture is very simple, fully flexible and cost effective. However, it suffers from high power losses and it also has a serious security threat.

Fully Static Architectures: Table 1(b) shows an example of RN1 of a fully static hybrid WDM/TDM PON architecture, referred to as a wavelength-split (WS) hybrid WDM/TDM PON [4]. In this architecture, each wavelength is routed to only one fixed TDM PON (or RN2). In a WS hybrid WDM/TDM PON, an AWG is put in RN1 to distribute different wavelengths to different TDM PONs. As an AWG has much less power loss compared to a power splitter, this architecture has a better power budget and can support more users and a longer reach. Moreover, it also has a high security. However, the flexibility is very restricted.

Partially Flexible Architectures: Table 1(c) shows an example of RN1 of a partially flexible hybrid WDM/TDM PON architecture. This category of architectures generally has a higher security and lower power loss than a fully flexible

TABLE I. REMOTE NODE 1 VARIANTS FOR (A) FULLY FLEXIBLE (B) FULLY STATIC AND (C) PARTIALLY FLEXIBLE HYBRID WDM/TDM PONS

\begin{tabular}{|c|c|c|c|}
\hline & $\begin{array}{c}\text { (a) B\&S RN1, } \\
\text { with power } \\
\text { splitter }\end{array}$ & $\begin{array}{c}\text { (b) WS RN1, } \\
\text { with AWG }\end{array}$ & $\begin{array}{c}\text { (c) ) B\&S RN1, } \\
\text { with power splitter } \\
\text { combined with WSS }\end{array}$ \\
\hline & & & \\
& & & \\
\hline Flexibility & Fully flexible & Fully static & Partially flexible \\
\hline Power loss & High & Low & Average \\
\hline Security & Low & High & Average \\
\hline
\end{tabular}

architecture, and is, of course, more flexible than a fully static architecture. In a partially flexible architecture, each TDM PON can be reached by multiple wavelengths and similarly each wavelength can reach multiple numbers of TDM PONs.

\section{EVALUATION APPROACH OF FLEXIBILITY IN HYBRID WDM/TDM PONS}

As discussed in section II, hybrid WDM/TDM PONs can have a different degree of flexibility. The more flexible architectures, however, are either more expensive, experience a higher power loss or are less secure. A question that arises is if a fully flexible architecture really needed, or if a partially flexible architecture can already serve similar flexibility advantages. An important assessment parameter for flexibility is the number of wavelengths required at a certain network load. If the number of wavelengths required for the same PON performance is less, the architecture will have less operational expenditures and a lower energy consumption as the more flexible architecture will use less wavelength line cards. The paper [3] gives a detailed overview of several flexibility advantages. In this section, we evaluate different architectural options for a hybrid WDM/TDM PON in terms of flexibility.

Architecture considered for evaluation: We simulate a hybrid WDM/TDM PON with $M=16$ TDM PONs, consisting of $N=4$ ONUs, and with 16 wavelengths with a capacity of 1 Gbps per wavelength (refer Fig. 1). We consider five different variations of a partially flexible hybrid WDM/TDM $\mathrm{PON}$, each with a varying degree of flexibility in RN1 by varying the values of $m_{s}$ and $m_{A W G}$ (or $m_{W S S}$ ), while keeping $M$ $=m_{s} \times m_{A W G}$ (or $m_{W S S}$ ) constant (cf. Fig. 2). Note that $m_{A W G}$ and $m_{W S S}$ represent the output port number of the AWG and WSS, respectively. We assume for our simulations, the values of $m_{A W G}$ or $m_{W S S}$ as $\{1,2,4,8,16\}$. All these configurations for the port number of WSS and AWG may not be the most relevant. However, for our simulation study and understanding the impact of flexibility, we feel that these values are most useful. For the different variants, Group $x$ indicates the number of TDM PONs $\left(x=m_{s}\right)$ that can share the same wavelength, or the number of wavelengths that can be used by one TDM PON (or RN2). For two extreme cases, this architecture is reduced to a fully flexible broadcast-and-select architecture $\left(m_{s}=16\right.$ or Group 16) and a fully static wavelength-split architecture $\left(m_{s}=1\right.$ or Group 1), respectively. The other architectures then represent the partially flexible architectures and specify that only 8,4 or 2 specific wavelengths (Group $8,4,2$ ) can be used by one TDM PON.

Traffic model: We study the performance of various variants of a flexible hybrid WDM/TDM PON architecture by using the OPNET simulation tool. In our model, we consider $R_{D}$ to be the data rate of the access link from a user to an $\mathrm{ONU}$, and $R_{U}$ to be the date rate of an upstream channel from an ONU to the OLT. The maximum distance between the OLT and ONUs is $100 \mathrm{~km}$. We choose, $R_{U}=1 \mathrm{~Gb} / \mathrm{s}$ and $R_{D}=R_{U} / N$ $=250 \mathrm{Mb} / \mathrm{s}$. We generate packets in the form of Ethernet frames ( 64 to1518 bytes) and packets arrive at each ONU from the end user. The simulated user traffic is self-similar by aggregating $S=32$ sub-streams [5], each consisting of 


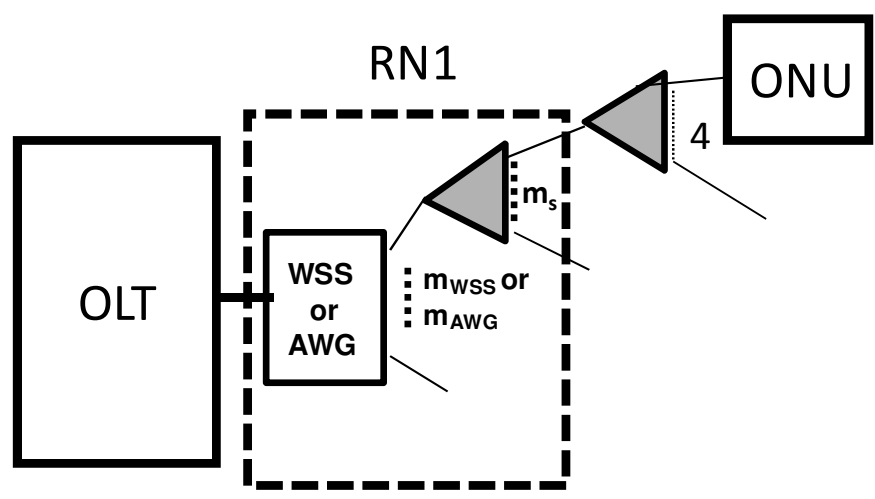

Figure 2: Hybrid WDM/TDM PON flavor based on AWG and power splitters considered for evaluation

alternating Pareto-distributed on/off periods, with a shape parameter of 1.4 for the on period and a shape parameter of 1.2 for the off period. In the on period, the packet arrivals are exponentially distributed with a mean arrival rate of $A_{r}$ (in $\mathrm{b} / \mathrm{s})$. The variable traffic load can be produced by varying $A_{r}$ and the location parameter for the on and off period. Different levels of burstiness can be achieved by varying $A_{r}$. To understand the effect of the traffic on the flexibility evaluation, we produce highly bursty and less bursty traffic. To produce highly bursty traffic, we use an $A_{r}$ value of $12.5 \mathrm{Mb} / \mathrm{s}$ for all loads. To produce less bursty traffic, we vary $A_{r}$ as $0.009 \Phi$, where $\Phi(\mathrm{Mb} / \mathrm{s})$ is the TDM PON Load. For less bursty traffic, on average, an ONU will be "ON" for more time.

A PON with long reach aggregates many users with different traffic profiles (such as business and home users) and over different geographical areas (such as rural and urban). The aggregation of self-similar traffic increases its burstiness [6] and the aggregation of users with different traffic profiles makes the traffic asymmetric. For example, the peak traffic hours of business and home users are at morning (near $10 \mathrm{am}$ ) and night (near $9 \mathrm{pm}$ ) respectively. Thus, a long reach PON with higher node consolidation has more bursty traffic. On the other hand, a short reach PON has more symmetric and less bursty traffic. However, to compare the effect of burstiness of traffic itself, we use the same reach $(100 \mathrm{~km})$ and number of users (64) for both less bursty and highly bursty traffic.

MAC protocol used: For evaluating different architectural options for a hybrid WDM/TDM PON, we use a medium access control (MAC) protocol [7] that exploits the offered flexibility in terms of dynamic wavelength allocation. Presently, we assume burst-by-burst switching for the WSS based configuration. A control signal from the OLT can be used to re-configure the WSS. The tuning and switching time of all components are neglected. We feel that the tuning and switching time of the components will lead to the same performance degradation for all RN2 configurations. Moreover, the performance degradation can be handled with a well suited MAC protocol, but this is out of the scope of this paper. The paper [8] discusses some of the ways to alleviate the performance degradation due to the tuning and switching time of the components.

\section{SIMULATION RESULTS}

As already mentioned, we have simulated five scenarios corresponding to different values of $m_{A W G}$ and $m_{s}$. The TDM PON load is normalized with a load of $1 \mathrm{Gbps}$. For our simulation, we first study the average number of wavelengths required as a function of the TDM PON load, and we show the average number of wavelengths required for different Group numbers. This study helps us to understand the advantage of grouping users and the benefits of multi-casting. Note that we measure the number of wavelengths required to provide services without any delay and loss to the users. This means that we allocate as many wavelengths to the TDM PON as is required by a TDM PON Group (without any restriction on the number of wavelengths). Our study will help operators to know the maximum number of wavelength line cards required for an optimum PON performance. Then we study the network performance for a given fixed number of line cards (in this case 16) at the OLT. Here we study how the use of an AWG based RN1 or a WSS based RN1 impacts the network performance. Obviously, the WSS based RN1 should have advantage over the AWG based RN1 due to its ability to dynamically distribute wavelengths. The network performance is measured in terms of NOF and packet delay. The network will be in an overloaded condition whenever the number of wavelengths required exceeds the number of wavelengths that can be allocated. We study the overloading condition in an AWG and WSS based RN1 with different RN2 configurations. For the AWG based RN1, an overloading condition occurs if the demand of a TDM PON exceeds the Group number whereas for the WSS based RN1, an overloading condition occurs whenever the demand of all TDM PON Groups exceeds the total available network resources. This study will help us to understand the advantage of switching and multicasting flexibility. Lastly, we also study the impact of switching and multi-casting flexibility on the packet delay.

First, we show the simulation results for a hybrid WDM/TDM PON with less bursty traffic and then with supposedly highly bursty traffic.

\section{A. Simulation Results for less bursty traffic}

We first show the average number of wavelengths required as a function of the TDM PON load. As already mentioned, the importance of such a graph is to see how many wavelength line cards are needed to achieve a high PON performance without any delay. For this study, the number of wavelengths required may not be bounded. Fig. 3 and Fig. 4 show the average and maximum number of wavelengths, respectively, required to satisfy the offered TDM PON (or RN2) load in the five considered scenarios. We see that for a load of 0.8 , a fully static solution will require about 16 line cards whereas a fully flexible solution will achieve the same performance with only 7 line cards. Thus, we can achieve more than 50\% power savings by employing a flexible solution. We notice a faster increase of the average number of wavelengths for Group 2 from a load of ca. 0.4. This can be explained as for Group 2, in the beginning, every TDM PON can typically be served by one wavelength, resulting in a total of eight required 


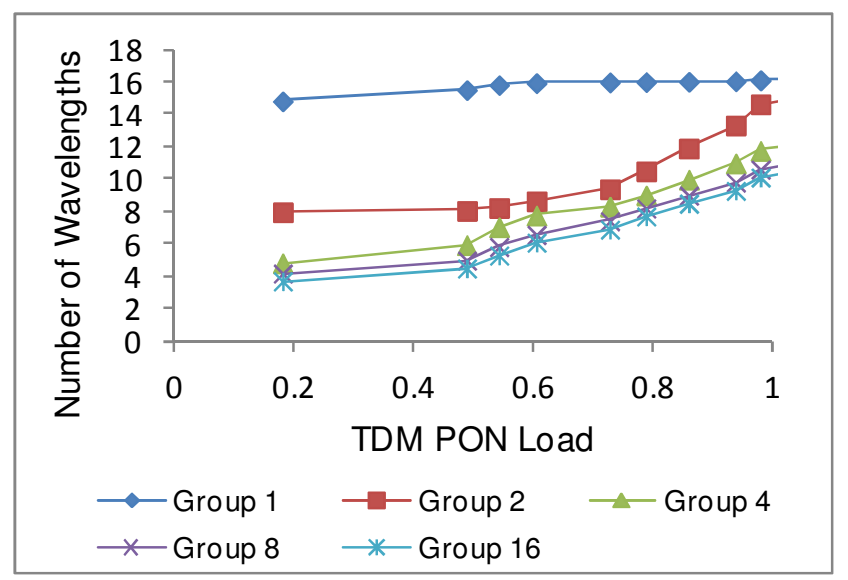

Figure 3: Average number of wavelengths required in function of the TDM PON or RN2 load, for five hybrid WDM/TDM PON variants with a different degree of flexibility in RN1 for less bursty traffic.

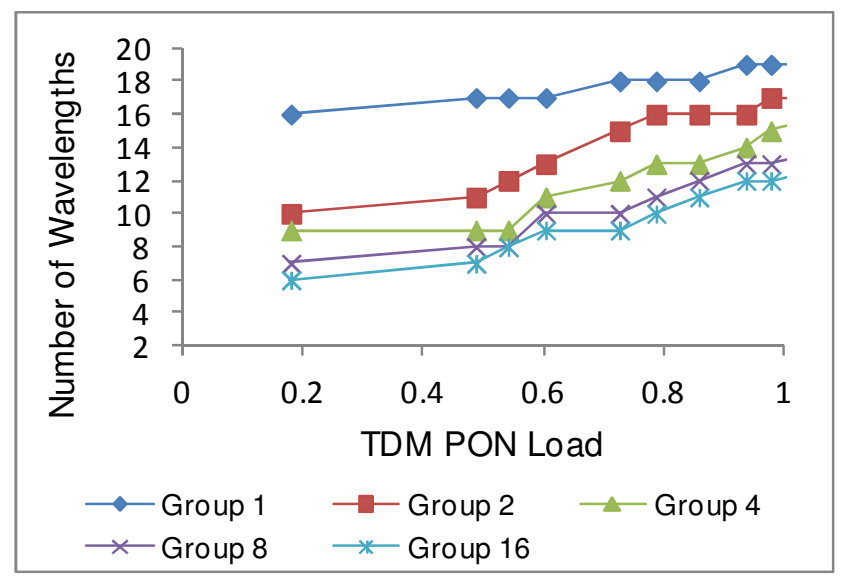

Figure 4: Maximum number of wavelengths required in function of the offered TDM PON or RN2 load, for five hybrid WDM/TDM PON variants with a different degree of flexibility in RN1 for less bursty traffic.

wavelengths. With higher loads, multiple (and not only one by one) TDM PONs will need a second wavelength, explaining the faster increase from that point. The simulation results clearly show that, already from the moment a certain degree of flexibility is available, large gains in terms of wavelength usage are possible, but from a given point the extra gain is very limited (the average number of wavelengths used from Group 4 to Group 16 is nearly the same).

We then evaluate the system performance with a restriction on the wavelength allocation. Whenever the system demands increases more than the system resources (which are in the present case 16 wavelengths) there will be an occurrence of an overloading situation. Fig. 5 gives the probability of an overloaded situation, expressed as NOF. The number of times a Group demands for more wavelengths than the Group number corresponds to an overloading situation as the channel capacity that is demanded is higher than the maximum channel capacity that can be allocated. We see that as the Group number increases, the probability of an overloaded situation decreases, since in a larger Group base we have the flexibility to multicast more wavelengths to each TDM PON Group. We

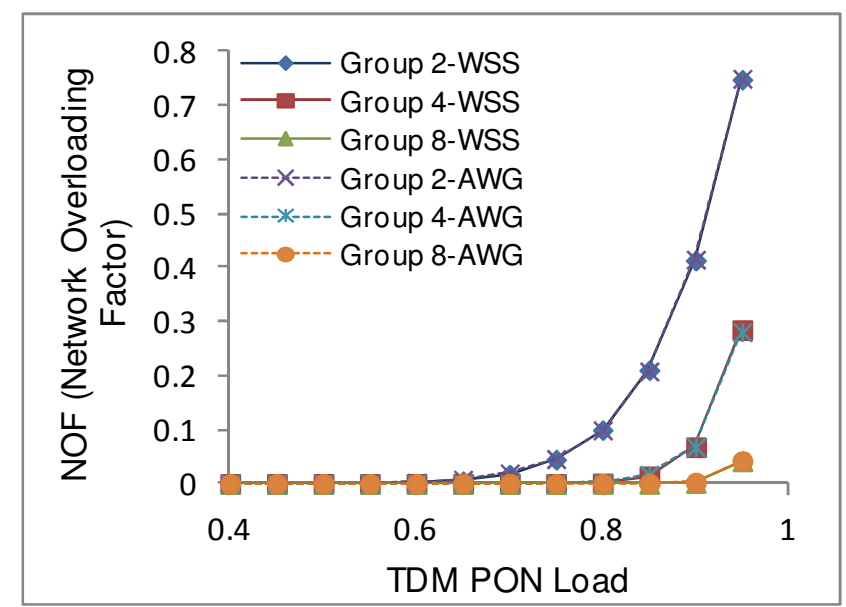

Figure 5: Probability of a overloaded situation in a TDM PON for varying load for WSS and AWG based configuration for less bursty traffic.

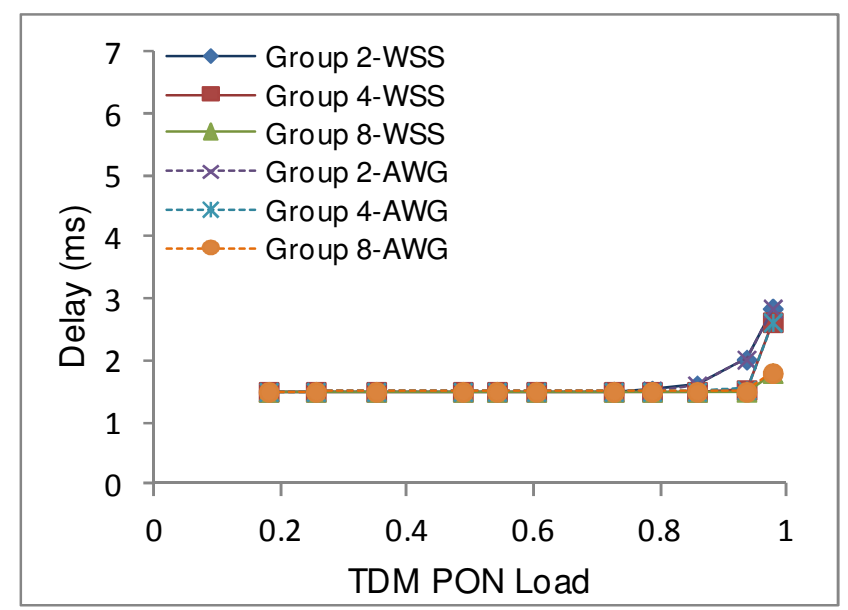

Figure 6: Delay vs load for hybrid WDM/TDM PON variants with a different degree of flexibility in WSS and AWG based RN1 configuration for less bursty traffic.

refer to this as multicasting gain. However, we see that WSS based hybrid WDM/TDM architectures do not improve the NOF significantly. As the traffic is less bursty, the demands of different Groups are nearly the same, and thus dynamic wavelength allocation does not give any clear benefit. Whenever there is an overloaded situation, the delay of the system will increase. Due to the same network overloading condition, both WSS and AWG based hybrid WDM/TDM PON architectures will also have the same delay performance (cf. Fig. 6). The lower bound of the delay equals $1.5 \mathrm{~ms}$ for the considered reach of $100 \mathrm{~km}$ (i.e. $3 / 2$ of the cycle time [9] or $3 / 2$ of the maximum round-trip time of the PON). The simulated traffic has a low peak-to-average load ratio of approximately 1.15 [i.e. $\left(A_{r} \times N \times S\right) / \Phi=0.009 \times 4 \times 32$ ], and thus the delay values are not very high at high load.

\section{B. Simulation Results for highly bursty traffic}

Here, we show the simulation results for highly bursty traffic, which is mainly noticed in long reach PONs. Fig. 7 and Fig. 8 show the average and maximum number of wavelengths, 


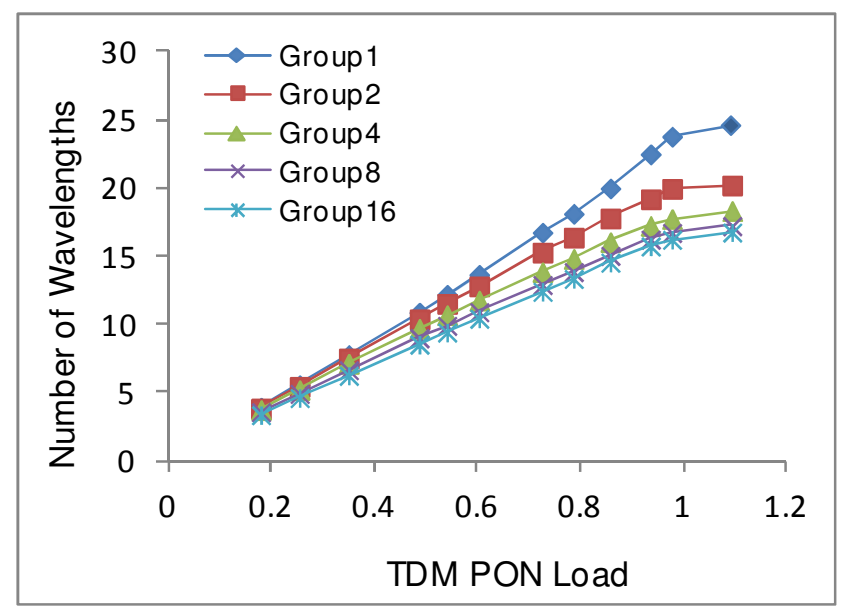

Figure 7: Average number of wavelengths required in function of the TDM PON or RN2 load, for five hybrid WDM/TDM PON variants with a different degree of flexibility in RN1 for highly bursty traffic.

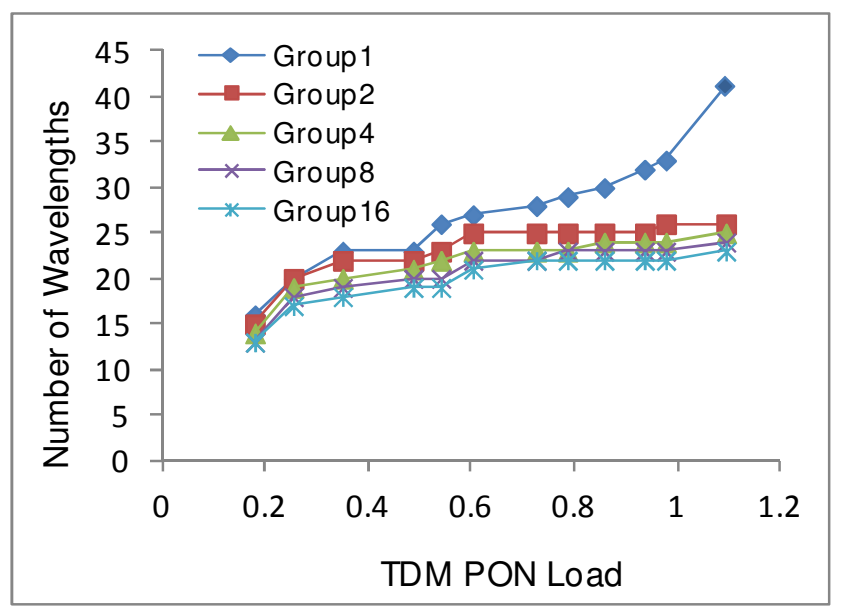

Figure 8: Maximum number of wavelengths required in function of the offered TDM PON or RN2 load, for five hybrid WDM/TDM PON variants with a different degree of flexibility in RN1 for highly bursty traffic.

respectively, required to satisfy the offered TDM PON (or RN2) load. There are many notable differences in the number of wavelengths required for highly bursty and less bursty traffic. First, we see that for highly bursty traffic at the low load, the number of wavelengths required for all Groups is almost the same in contrast to the less bursty traffic where there is a significant difference in the number of wavelengths required for Group 1 (on an average three wavelengths) and Group 16 (on an average 15 wavelengths). This can be explained as in the bursty traffic, a user is "ON" for a smaller period and thus the probability of two users to be "ON" at the same time will be even less. Thus, at the low load condition, when the probability of a user to be in active state is less, grouping of users will not make any difference in the wavelength consumption as most of the time only one user of the Group will be in "ON" state. However, if the probability of a user to be "ON" is high (at high load), multicasting will have an impact. Second, we also see that at low load (0.2), the number of wavelengths required for Group 16 is only four

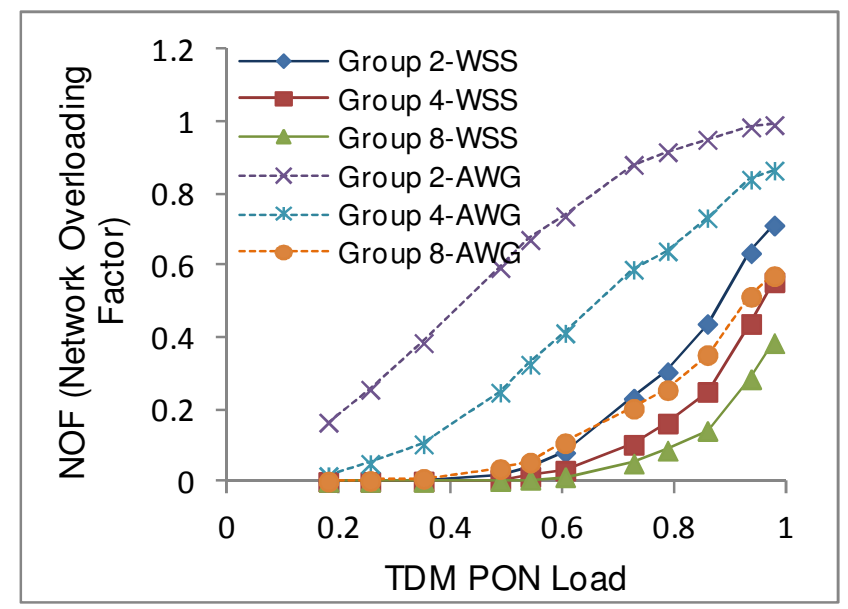

Figure 9: Probability of a overloaded situation in a TDM PON for varying load for WSS and AWG based configuration for highly bursty traffic.

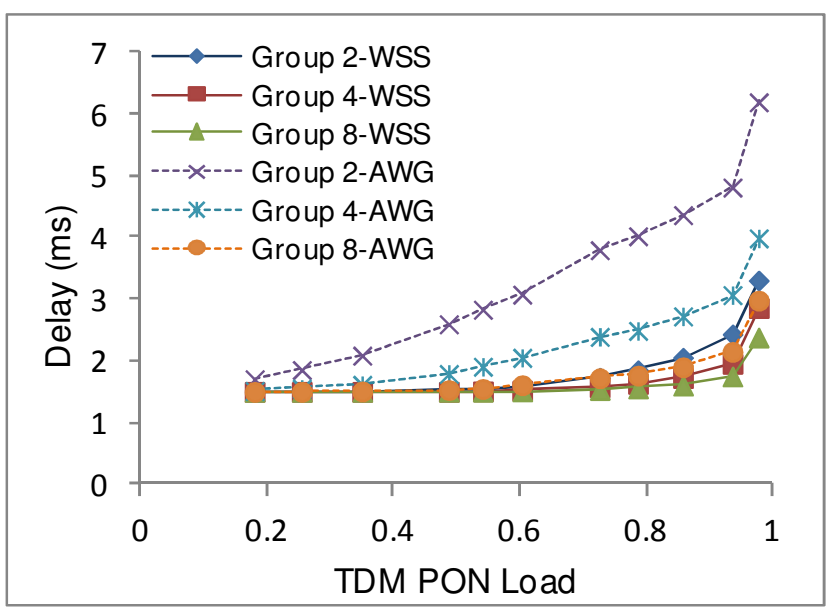

Figure 10: Delay Vs load for hybrid WDM/TDM PON variants with a different degree of flexibility in WSS and AWG based RN1 configuration for highly bursty traffic.

whereas for less bursty traffic, Group 16 requires on average 15 wavelengths. This can again be explained on the basis of the traffic burstiness. For less bursty traffic, a user is usually in "ON" state, and this increases the wavelength usage. However, for highly bursty traffic, a user is "ON" for a smaller period, and there may be many periods in which it requires no single wavelength. Then, we investigate the influence of using a WSS and AWG based RN1. Fig. 9 gives the probability of an overloaded situation for both RN1 configurations. We can clearly see that the hybrid WDM/TDM PON employing WSS at RN1 is less overloaded as compared to the static hybrid WDM/TDM PON with AWG at RN1. When the traffic is bursty, the user demands vary significantly in a Group and a WSS can dynamically allocate wavelengths according to the instantaneous Group requirements. Thus, for a more bursty traffic scenario (which will be the case with higher node consolidation), the dynamic wavelength configuration that is possible with WSS provides significant advantages. We see that the configuration with WSS and Group 2 has less overloading probability than the configuration with an AWG 
and Group 4. The result shows that we can improve the flexibility of a PON architecture by adding an active remote node even with reduced power splitting. Moreover, lower values of NOF will lead to the low delay values as can be seen in Fig. 10. From the above results, it is clear that as the nextgeneration PONs aims for a higher reach, the WSS based configurations can give significant flexibility advantages. The availability of 1:23 port WSS with a low insertion loss [10] makes it even more promising for the next-generation PON solutions.

\section{CONCLUSION}

In this paper we have evaluated through simulation the gains of using flexible architectures in next-generation optical access (NGOA) networks, and more specifically in hybrid WDM/TDM PONs. In particular, we have evaluated two types of flexibility gains, due to the multicasting and switching functionality, respectively. We conclude that a partially flexible network can already provide enough multicasting gain compared to a fully flexible network. Moreover, we have shown through simulations that in long reach PON, where the traffic can be highly bursty, a partially flexible network may further benefit from the switching flexibility which can be delivered by a reconfigurable switch like wavelength selective switches (WSSs). However, for short reach PON solutions, due to presumably less bursty traffic, switching functionality will not lead to any significant advantages in PON performance. Thus, if an operator is aiming for higher reaches, then using a reconfigurable remote node solution looks like a promising one. However, for short reach PON solutions with less customer aggregation, reconfigurability at the remote node may not be the way forward. Our study will help network operators and service providers to choose the most suitable architecture for NGOA networks.

\section{ACKNOWLEDGMENT}

The research leading to these results has received funding from the European Community's Seventh Framework Programme (FP7/2007-2013) under grant agreement $\mathrm{n}^{\circ}$ 249025 (ICT-OASE).

\section{REFERENCES}

[1] 249025-ICT OASE Project, Requirements for European NextGeneration Optical Access Networks, D2.1.

[2] G. Das et al., "A Hybrid WDM/TDM PON Architecture Using Wavelength Selective Switches", 4th IEEE International Symposium on Advanced Networks and Telecommunication Systems (ANTS 2010), Mumbai, India, Dec. 16-18, 2010.

[3] A. Dixit et al., "Evaluation of flexibility in hybrid WDM/TDM PONs", 1st International Symposium on Access Spaces (IEEE-ISAS2011), Yokohama, Japan, June 17-19, 2011.

[4] C. Bock, J. Prat, and S. D. Walker, "Hybrid WDM/TDM PON Using the AWG FSR and Featuring Centralized Light Generation and Dynamic Bandwidth Allocation", Journal of Lightwave Technology, vol. 23, no. 12, Dec. 2005, pp. 3981-3988.

[5] W. Willinger et al., "Self-similarity through high variability: statistical analysis of Ethernet LAN traffic at the source level", IEEE/ACM Transactions on Networking, vol. 5, no. 1, Feb. 1997, pp. 71-86.
[6] W.E Leland et al., "On the self-similar nature of Ethernet traffic", IEEE/ACM Transactions on Networking, vol. 2, no. 1, Feb. 1994, pp. 115.

[7] G. Das et al., "A New Architecture and MAC Protocol for Fully Flexible Hybrid WDM/TDM PON", Proc. of ECOC 2009, Vienna, Austria, Sep. 20 - 24, 2009, paper P6.28.

[8] J. Zhang, and N. Ansari, "Scheduling Hybrid WDM/TDM Passive Optical Networks with Nonzero Laser Tuning Time", IEEE/ACM Transactions on Networking, vol. 19, no. 4, Aug. 2011, pp 1014 - 1027.

[9] B. Lannoo et al., "Analytical Model for the IPACT Dynamic Bandwidth Allocation Algorithm for EPONs", Journal of Optical Networking, vol. 6, no. 6, Jun. 2007, pp. 677-688.

[10] Available at :

http://www.ofcnfoec.org/osa.ofc/media/Default/PDF/2009/09-Poole.pdf 